\title{
Prescription Pattern of Long Acting Haloperidol in a Tertiary Psychiatric Institution in South India: Ten - Year Retrospective Records Study
}

Original Article

Sudha Srinivasaragavan, Poornachandrika P

- Institute of Mental Health, Chennai

Vijaya Raghavan

- Schizophrenia Research Foundation

\section{Abstract}

\section{BACKGROUND}

Long acting antipsychotics injections (LAIs) have proven efficacious with regard to improvement in symptoms, adherence and reduction of caregiver burden. Yet, their prescription pattern is less studied. Hence, the aim of the study was to examine the prescription pattern of a long acting antipsychotic injection, haloperidol, in a large tertiary psychiatry hospital over a period of 10 years from 2009 to 2018.

\section{MATERIALS AND METHODS}

Retrospective study design was used to collect data for a time period of 10 years from $2009-2018$. The data were collected from the hospital records for the total number of persons with mental health disorders used the tertiary center and from the central pharmacy records on the total long acting antipsychotics injection (Haloperidol LAI) dispensed during the study period.

\section{RESULTS}

Despite unchanging patient inflow, there was a decline in utilization of long acting haloperidol injection. The average number of haloperidol units utilized was $1595.5 \pm 294.8$. The use sloped down from an average of 16 units per 1000 patients per month in 2009 to about 9 units per 1000 patients per month in 2018.

\section{CONCLUSION}

Steady declining pattern in the prescription of LAIs for the management of mental health disorders is observed, even though they are proven to be effective and have ease of use. It is important to explore the reasons and provide education and training for better utilization of LAIs among mental health professionals.

\section{Corresponding author:}

Dr. Vijaya Raghavan

Schizophrenia Research Foundation,

R/7A, North Main Road, Anna Nagar West Extension, Chennai, Tamilnadu - 600101.

Email: vijayaraghavan@scarfindia.org

\section{Keywords: haloperidol, long acting injections, prescription pattern}

\section{Running title: Prescription Pattern of LAI Haloperidol in South India}




\section{INTRODUCTION}

Schizophrenia is one of the mental disorders that presents with a chronic, usually unremitting, progressive course in the absence of medical intervention. According to the National Mental Health Survey of India (2015-16), schizophrenia spectrum disorders amount to lifetime morbidity in $1.4 \%$ of the population in India. ${ }^{(1)}$ Patients with schizophrenia usually present with positive symptoms (hallucinations and delusions), negative symptoms (apathy, amotivation and anhedonia) and cognitive symptoms (attention deficit, problem solving and memory). Schizophrenia can lead to burden to the patients and their caregivers leading to reduced quality of life.

Antipsychotic drugs are the mainstay of treatment in schizophrenia spectrum disorders. Antipsychotic drugs are available in the form of oral and injectable preparations. Long acting antipsychotics injections (LAIs) have proven efficacious with regard to improvement in symptoms, adherence and reduction of caregiver burden. ${ }^{(2-5)}$ Standard guidelines are available regarding the use of LAIs. ${ }^{(6-8)}$ LAIs have also been used in relapse prevention in first episode psychosis and in maintenance therapy for affective psychoses. Long acting injections are one of the modes of management of non-compliance with psychiatric drugs, which ranges from $10-77 \% .{ }^{(9)}$ The use of long acting antipsychotics has been widely studied all over the world. The use of LAIs are substantially lower when compared to the use of oral antipsychotics. ${ }^{(10)}$ Various patient, psychiatrist and mental health service-related factors could contribute to this discrepancy. Very few studies have explored the utilization of the LAIs in the Indian subcontinent. It is important to understand the changing patterns in the utilization of LAIs over a period in time to understand the pattern and this would help us understand the reasons for the underutilization of LAIs in India. Hence, the aim of the study was to examine the cumulative utilization of LAIs in our large tertiary mental health institution in South India over the past 10 years.

\section{MATERIALS AND METHODS}

The study was conducted at the Institute of Mental Health, Madras Medical College, Chennai. Institute of Mental Health is one of the largest tertiary care mental health institution in South India providing mental health services to Tamil Nadu and neighboring states. Institutional ethics committee approval was obtained before the start of the study. The study was conducted in the month of June - August 2019.

A retrospective study design was used to collect data for a time period of 10 years from $2009-2018$. The sources of data regarding the utilization of LAIs were the audited central pharmacy records and the yearly drug account book from the outpatient department. The validity of the data collected from these records were ascertained by the senior administrators. Yearly utilization of LAIs as a whole was collected from these records and was cross verified. Any discrepancy between the records was discussed with the concerned authorities and with senior administrators to reach a consensus. For this study, only the number of haloperidol decanoate long acting injection units (One Unit is an $1 \mathrm{ml}$ ampoule containing $50 \mathrm{mg} / \mathrm{mL}$ of haloperidol decanoate) utilized in each year were collected. There was no change in the definition or composition of one unit in the study period. The flowchart of the research methodology followed is depicted in Fig 1.

Fig 1. Overall research methodology of data collection
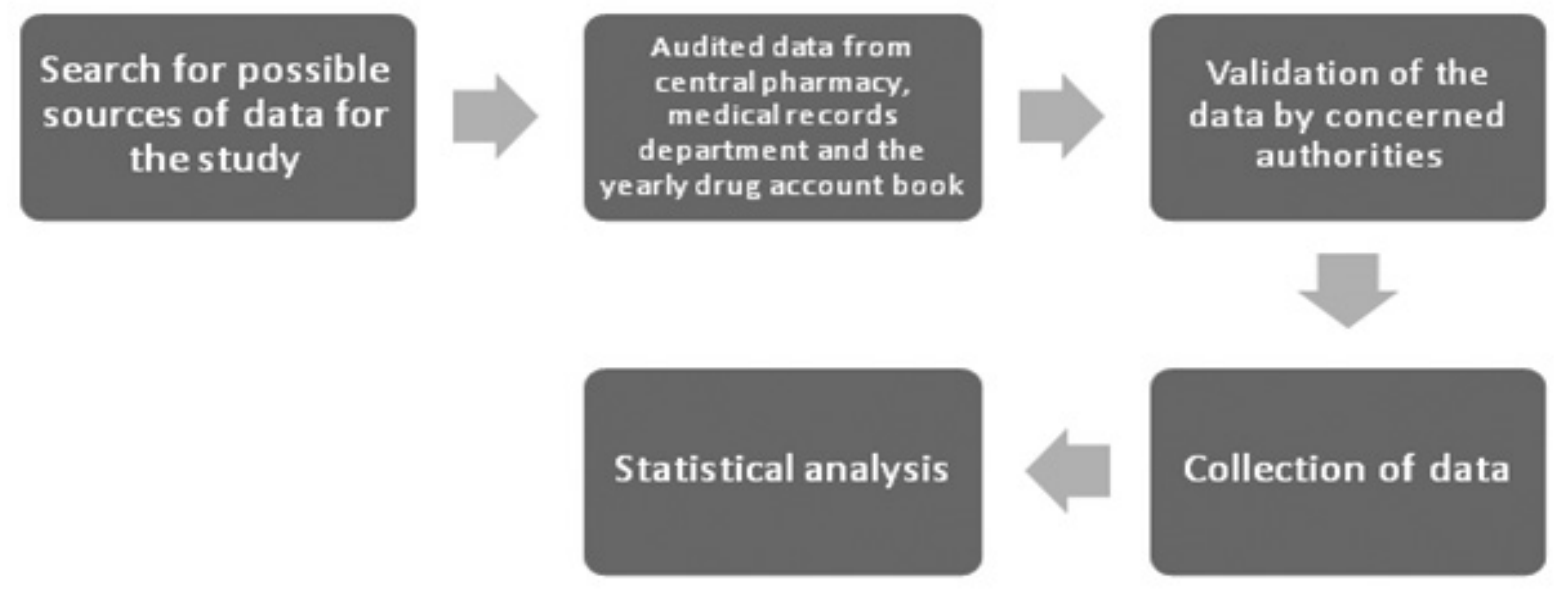


\section{Statistical Analysis}

All the statistical analysis was done using SPSS 16.0. Descriptive statistics were used to analyze the data. Time series analysis and forecasting using Holt modelling was done to predict the future patterns in the utilization of haloperidol LAIs.

\section{RESULTS}

Results indicated a steady decline in the utilization of long acting Haloperidol injection, despite nearly the unchanging patient inflow (Mean $=130007 \pm$ SD 7829) in the 10 year period of study. The average number of haloperidol LAIs utilized was $1595.5 \pm 294.8$ per year. The use sloped down from an average of 16 units per 1000 patients per month in 2009 to about 9 units per 1000 patients per month in 2018 (Table 1).

Table 1. Summary of the total census in the institution and number of haloperidol units utilized per year for the years 2009 - 2018

\begin{tabular}{|c|c|c|}
\hline Year & OPD census & Total Number of Haloperidol Units Utilized \\
\hline 2009 & 121907 & 1916 \\
\hline 2010 & 127919 & 2174 \\
\hline 2011 & 139771 & 1363 \\
\hline 2012 & 139110 & 1535 \\
\hline 2013 & 133125 & 1660 \\
\hline 2014 & 141735 & 1664 \\
\hline 2015 & 121411 & 1436 \\
\hline 2016 & 123743 & 1681 \\
\hline 2017 & 123999 & 1373 \\
\hline 2018 & 127354 & 1153 \\
\hline
\end{tabular}

Fig 2. Predicted pattern of the utilization of long acting haloperidol using time series analysis

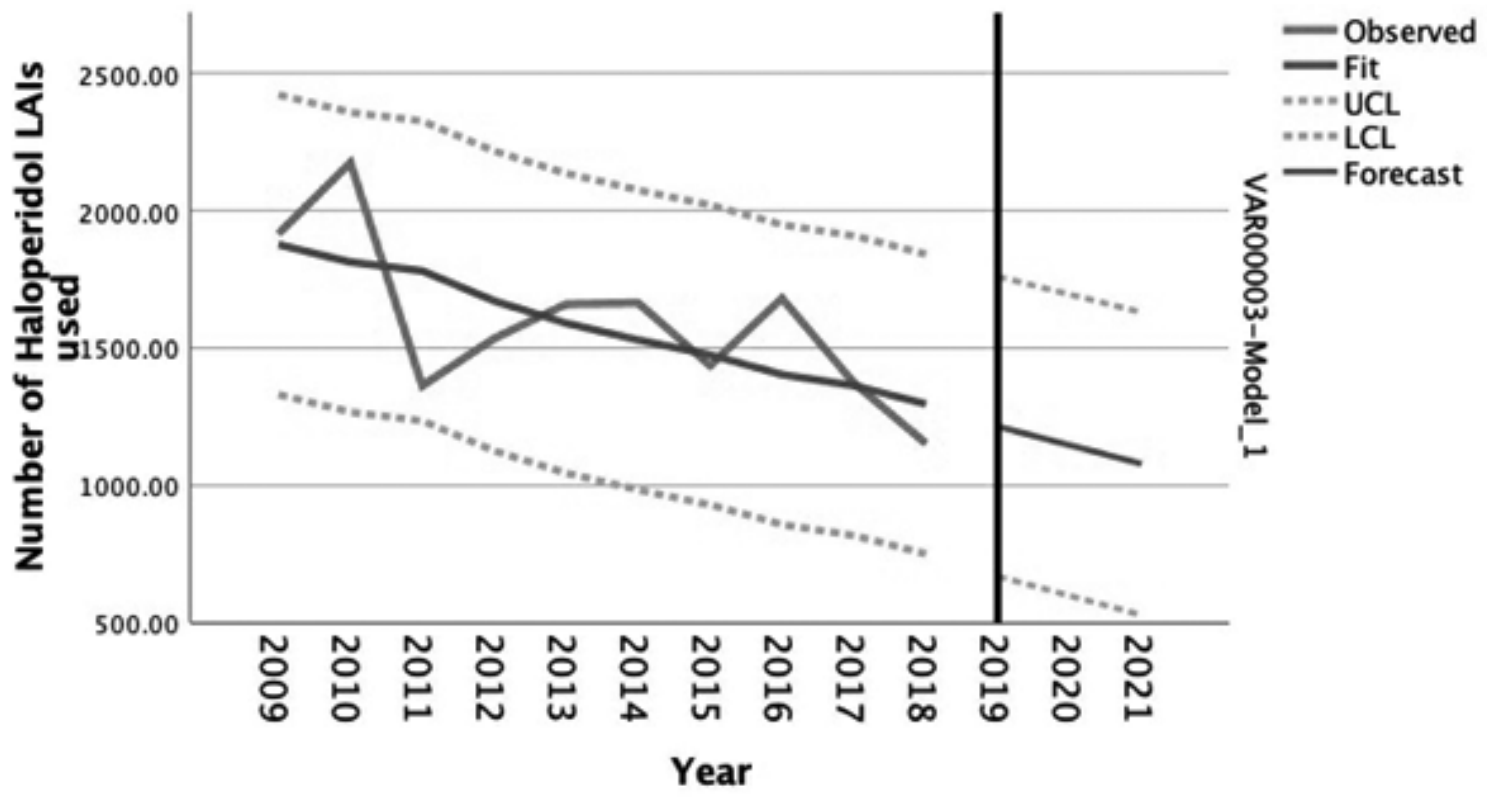

The time series analysis of the data and forecasting of the haloperidol LAIs is depicted in the Fig 2. The forecasting analysis indicated a progressive fall in the utilization of haloperidol LAIs for the years 2020-2022. 


\section{DISCUSSION}

The aim of the study was to assess the utilization patterns of haloperidol LAIs in a large tertiary mental health institution in the last 10 years using the audited hospital records. The results indicate that there is a steady declining pattern in the prescription of LAIs for the management of psychotic disorders, even though they are proven to be effective and have ease of use. Similar patterns of declining utilization of LAIs has been observed in other studies from other parts of the world. ${ }^{(10)}$ This is despite high prevalence $(10-77 \%)$ of medication non-adherence among patients with psychotic disorders. $^{(11)}$

Various reasons could be speculated for this decline in the use of LAIs in the clinical practice. Reasons could include the availability of better oral antipsychotics; and client, and clinician attitude towards LAIs and lack of training and fear among the younger generation of psychiatrists towards the use of LAIs. ${ }^{(12)}$ There is a general belief that injectable drugs are for the non-compliant patient even though they are a better choice in first episode psychosis and LAIs have a better continuation rate when compared to oral antipsychotics. ${ }^{(13)}$

Literature also points to patient experience with injectable drugs as being poor as they feel injections shift the control over therapy from patients to the physician. (14) In general, LAIs have been looked at with some negativity in psychiatric practice. There is a presumption that the introduction of newer antipsychotics plays a role in the decline of FGA LAI utilization, probably replacing them in various instances, avoiding the unpleasant side effects associated with FGA LAIs.

Though the aspect of cost of LAIs is not studied in the current study as it was conducted in a public funded hospital, cost of LAIs has been a major factor behind their utilization in routine clinical practices in other settings. ${ }^{(15)}$ Long acting antipsychotic injections have a similar side effect profile when compared to oral antipsychotics. ${ }^{(16)}$ Substantial training of psychiatrists in the utilization of LAIs is pivotal to the judicious use of the same, the lack of which might be a key factor behind the reluctance to free usage of LAIs. The general awareness of treating psychiatrists to the utility of LAIs is affected by various factors from training to availability of the particular drug to his experience with the same. There are even reports of doctors' belief about patient perception of LAIs as being negative assumptions: that a patient would refuse an injectable drug or presenting an injectable would strain the therapeutic relationship ${ }^{(17)}$ in spite of evidence to the contrary. ${ }^{(18)}$

This study is not without any limitations. Since this study was a central hospital record-based study, the same cannot be extrapolated on the utilization patterns among the other mental health settings such as private clinics and general hospital psychiatry units. specific details regarding indications, dosing and duration in each patient cannot be arrived at in this study. This poses the difficulty of examining for which disorder the haloperidol LAIs have been prescribed. There were short periods of "out of stock" for haloperidol decanoate in the study site, which could decrease the estimate of utilization. Data on cumulative utilization of the other long acting injection Fluphenazine decanoate was not included, as it has been used sparsely in the past ten years. Other long acting injection preparations of risperidone were not included as they are not procured at the study site. Also, since the study includes the cumulative census of all the patients seeking help without regards to diagnosis, there seems to be an underestimation of utilization, as most of the LAIs are prescribed for patients with psychotic disorders and not others. Nevertheless, there is a wide gap in LAI utilization when compared to reports from other countries. This is projected to be $1.5 \%-1 \%$ of all patients whereas, a study from Europe shows a utilization rate of $25 \%$ in psychotic disorders. ${ }^{(19)}$

\section{CONCLUSION}

The results indicate that there is evidence to the declining pattern towards the utilization of haloperidol LAIs. It is important to understand the various reasons contributing to this and efforts should be made to encourage the use of LAIs wherever indicated in the routine clinical practice. More research is required regarding physician and patient attitude towards LAIs and finding ways to improve efficient and adequate utilization of LAIs, such as workshops on the practice of prescribing LAIs for clinicians, young psychiatrists and residents.

\section{ACKNOWLEDGEMENT}

Authors express their sincere thanks to all the Records Department staff for their expertise, time and energy in searching, retrieving and organizing all the relevant records and information needed for this study. 
Nil

SOURCE OF FUNDING

Nil

\section{References}

1 Gururaj G, Varghese M, Benegal V, Rao G, Pathak K, Singh L, et al. National Mental Health Survey of India, 2015-16: prevalence, patterns and outcomes. Bengaluru: National Institute of Mental Health and Neuro Sciences, NIMHANS Publication. 2016;129.

2 Kaplan G, Casoy J, Zummo J. Impact of long-acting injectable antipsychotics on medication adherence and clinical, functional, and economic outcomes of schizophrenia. Patient Preference and Adherence. 2013;7:1171-1180.

3 Marcus SC, Zummo J, Pettit AR, Stoddard J, Doshi JA. Antipsychotic adherence and rehospitalization in schizophrenia patients receiving oral versus long-acting injectable antipsychotics following hospital discharge. Journal of Managed Care \& Specialty Pharmacy. 2015;21(9):754-769.

4 Miyamoto S, Fleischhacker WW. The use of long-acting injectable antipsychotics in schizophrenia. Current Treatment Options in Psychiatry. 2017;4(2):117-126.

5 Park SC, Choi MY, Choi J, Park E, Tchoe HJ, Suh JK, et al. Comparative efficacy and safety of long-acting injectable and oral second-generation antipsychotics for the treatment of schizophrenia: a systematic review and meta-analysis. Clinical Psychopharmacology and Neuroscience. 2018;16(4):361-375.

6 Chou YH, Chu PC, Wu SW, Lee JC, Lee YH, Sun IW, et al. A systemic review and experts' consensus for long-acting injectable antipsychotics in bipolar disorder. Clinical Psychopharmacology and Neuroscience. 2015;13(2):121-128.

7 Llorca PM, Abbar M, Courtet P, Guillaume S, Lancrenon S, Samalin L. Guidelines for the use and management of long-acting injectable antipsychotics in serious mental illness. BMC Psychiatry. 2013;13(1):340

8 McGorry P, Killackey E, Lambert T, Lambert M, Jackson H, Codyre D. Royal Australian and New Zealand College of Psychiatrists clinical practice guidelines for the treatment of schizophrenia and related disorders. Australian and New Zealand Journal of Psychiatry. 2005;39(1-2):1-30.

9 Lucca JM, Ramesh M, Parthasarathi G, Ram D. Incidence and factors associated with medication nonadherence in patients with mental illness: a cross-sectional study. Journal of Postgraduate Medicine. 2015;61(4):251.

10 Patel MX, Taylor M, David AS. Antipsychotic long-acting injections: mind the gap. The British Journal of Psychiatry. 2009;195(S52):S1-S4.

11 Raghavan V, Mohan G, Gopal S, Ramamurthy M, Rangaswamy T. Medication adherence in first-episode psychosis and its association with psychopathology. Indian Journal of Psychiatry. 2019;61(4):342.

12 Parellada E, Bioque M. Barriers to the use of long-acting injectable antipsychotics in the management of schizophrenia. CNS Drugs. 2016;30(8):689-701.

13 Jeong HG, Lee MS. Long-acting injectable antipsychotics in first-episode schizophrenia. Clinical Psychopharmacology and Neuroscience. 2013;11(1):1.

14 Potkin S, Bera R, Zubek D, Lau G. Patient and prescriber perspectives on long-acting injectable (LAI) antipsychotics and analysis of in-office discussion regarding LAI treatment for schizophrenia. BMC Psychiatry. 2013;13(1):261. 
15 Kim SW, Lee YH, Jang JE, Yoo T, Kim JM, Shin IS, et al. Comparison of attitudes toward long-acting injectable antipsychotics among psychiatrists and patients. International Clinical Psychopharmacology. 2013;28(2):80-86.

16 Kane JM, Kishimoto T, Correll CU. Assessing the comparative effectiveness of long-acting injectable vs. oral antipsychotic medications in the prevention of relapse provides a case study in comparative effectiveness research in psychiatry. Journal of Clinical Epidemiology. 2013;66(8):S37-S41.

17 Iyer S, Banks N, Roy M-A, Tibbo P, Williams R, Manchanda R, et al. A qualitative study of experiences with and perceptions regarding long-acting injectable antipsychotics: part I-patient perspectives. The Canadian Journal of Psychiatry. 2013;58(5_Suppl):14-22.

18 Walburn J, Gray R, Gournay K, Quraishi S, David AS. Systematic review of patient and nurse attitudes to depot antipsychotic medication. The British Journal of Psychiatry. 2001;179(4):300-307.

19 Barnes T, Shingleton-Smith A, Paton C. Treatment of schizophrenia by long-acting depot injections in the UK. The British Journal of Psychiatry. 2009;195(52):S37-S42. 